\title{
Processing and Unification of Environmental Noise Data from Road Traffic with Spatial Dimension Collected through Mobile Phones
}

\author{
Petr Duda \\ Laboratory on Geoinformatics and Cartography, Masaryk University, Brno, Czech Republic \\ Email: 147169@mail.muni.cz
}

How to cite this paper: Duda, P. (2016) Processing and Unification of Environmental Noise Data from Road Traffic with Spatial Dimension Collected through Mobile Phones. Journal of Geoscience and Environment Protection, 4, 1-26.

http://dx.doi.org/10.4236/gep.2016.413001

Received: July 21, 2016

Accepted: December 24, 2016

Published: December 27, 2016

Copyright $\odot 2016$ by author and Scientific Research Publishing Inc. This work is licensed under the Creative Commons Attribution International License (CC BY 4.0).

http://creativecommons.org/licenses/by/4.0/

\begin{abstract}
Noise measurement using mobile phones is now developed very well. While there are some good applications for the measurement of noise from road traffic, thus on processing of measured data is only paid a very little attention. The data, however, are burdened by specific errors and for further work with them it is necessary to adjust and determine their uncertainty. One of the biggest problems is inaccuracy in position versus the noise source and the shortest length of measurement that can be regarded as representative. Imprecision in terms of location can be determined by calculating the variance of possible distance from the noise source, which for measurement of traffic noise requires a map-matching data points both transverse to the street (sidewalk) network and in the longwise direction. During typical urban measurements, this error can even reach $7-10 \mathrm{~dB}$. Three basic types of algorithms for the calculation of uncertainty and positional correction based on the type of input and output data (raster, vector, vector-oriented) were tested. Uncertainty in the variability of the measurement data is necessary to determine from the number of passing vehicles per time unit. The presented solutions are implemented in the Mobile Noise system.
\end{abstract}

\section{Keywords}

Measurement Uncertainty, Environmental Noise, Mobile Phone, Citizen Science, Noise Mapping

\section{Crowdsourcing of Environmental Noise Measurement}

Currently, there is a massive expansion of a new phenomenon: use of mobile 
phones for data collection and then voluntary collection and processing of the data using a variety of social networks. This concept can be applied among others, also to the problem of environmental noise pollution. Although it has a number of limitations, as demonstrated by first studies (e.g. [1] [2]), it may successfully act as an appropriate complementary tool for monitoring and assessment of the damage caused by excessive noise.

Over the past 10 years, several projects in the field of community noise mapping have been created. These projects are primarily trying to introduce noise mapping technology using mobile phones and processing of data acquired by this way. Albeit, relatively large amount of data have been collected by these studies. Further use of these data still remains a big question. These studies were primarily aimed to test the concept of noise data crowdsourcing, while they did not develop issues of their further processing and use. This kind of data, if they are published at all, most often rely on the fact that they will be used in their raw form. This approach significantly reduces usefulness of the data for further analysis and evaluation of noise situation. This article primarily focuses on post processing of the noise data on example of ground transportation noise data from the point of view of the uncertainties in the positioning and in terms of changes in land transport operations during daytime.

\subsection{Smartphone Applications for Crowdsourced Noise Measurement}

The work of Santini et al. [3] was one of the first to use mobile phones to measure environmental noise. Authors particularly examine technical problems that affect the design and implementation of systems which use mobile phones to assess noise pollution. This paper is not yet focused on the development of the entire system and noise maps cannot be created from presented incomplete and random sampling. Later, e.g. at work [4] authors presents full system for noise mapping, but this system lacks A weighting filtering as input procedure for obtain the equivalent noise levels, so it is not possible to compare outputs with standard noise maps.

The most important systems able to map noise using smartphones as sensors, however, are these:

- NoiseSpy [5] - This application is one of the first which allows collection of acoustic noise levels data using mobile phones. The work focuses mainly on to the measurement itself and also on quality of measured data. These data can be processed using GIS tools and displayed e.g. using Google Maps. Because of great positional uncertainties of the measurement, Authors visualize the results on a web-based Google Maps map using regular square matrix with a cell size bigger than $20 \times 20$ meters. Point measurements can be exported to Google Earth, where they are displayed semi-transparent colored circles with a color corresponding to the noise level according to the color 
spectrum. Data are not adjusted in any way. Application and data were never released to the public.

- NoiseDroid [6]-Application allowing collection of data on noise using mobile phones with the Android operating system. It supports manual, automatic, event or series measurements and visualizes all the collected measurements as a table or a map. Data can be viewed and filtered by various criteria. Users can export measurement results to the Open Noise Map web portal and they are also able to import measurements from this community server. Source code is available, but the application itself is no longer being developed and maintained. There is, or should be, an interface for Google Maps, Open Street Maps, Bing Maps and Ovi Maps. Map visualizes the noise level by so-called heat map, data are clustered using the DBSCAN algorithm [7]. Again, there is complete lack of any further data processing.

- NoiseMap [8] - This is a noise map from mobile phones sensors in history and in real time. It is a part of the project for visualization of data from various sensors using da_sense.de software. This map is visualized using a hexagonal grid, where color scale shows the noise level. A coupled mobile application for data measurement called with same name is presenting also a frequency calibration and incentive mechanisms (gaining points for success measurements) and is downloadable for Android platforms. The source code is currently not accessible.

- AirCasting [9] - Initiative of a non-governmental organization based in New York [10]. They, in cooperation with a Polish subcontractor, developed an application for Android, which allows to collect data on noise. Web portal allows to visualize also a large number of other parameters from other types of sensors (dust, moisture levels of $\mathrm{CO}, \mathrm{CO}_{2}$, etc.). Wherein there is no sensor classification, it is very difficult to search, view and process appropriate data type. Users can annotate data by text, signs and photographs. Both the mobile application and the Web platform are open source. In the mobile application, users can calibrate the input using a trim application method. On the web portal, data are dynamically clustered into a square grid and using this grid an average values are calculated for a every grid cell with measurements. However, this application lacks measuring accuracy specification.

- WideNoise [11]-An application developed for mobile phones with operating systems iOS and Android by a company called Wide Tag [12]. This application is a representative of a number of similar applications that measure noise level only for a short period after button pressing (in this case 5 seconds) and are designed to measure noise levels in certain situations to help users interpret the value of the noise levels in these cases (e.g. sleeping cat emits a sound on level of about 40 to $50 \mathrm{~dB}$, the passage of typical passenger car ranges from 70 to $80 \mathrm{~dB}$, etc.). Measurements can be marked by coordinates and may be published on social networks or on the WideNoise 
website, where they are displayed on a map. In 2011, based on the project Every Aware [11], an updated version 3.0 was processed, where also annotations are available. Source code of WideNoise v3.0 for iOS and Android is available under open source license. WideNoise uses linear interpolation to compensate microphone sensitivity etc. It is not possible to calibrate the application for different types of devices.

- Ear-Phone [13] - This project presents a general solution for monitoring environmental noise using mobile phones. The main task of this system is find how to solve the fundamental problem to get noise maps from incomplete and random samples obtained by crowdsourcing data collection. Different interpolation and classification methods are used for achieving this target. Ear-Phone, which is implemented on the Nokia N95 and N97, HP iPAQ and HTC One, also addresses the question of the measurement precision and data of noise pollution collection on the mobile device. One of the biggest problems using smart phones as sensors is that the results of the sensor measurements may vary depending on the orientation of the phone and the user's context (for example, if the user carries the phone in a bag or hold it in your hand). To solve this problem, Ear-Phone introduces detection of device relative position context, and application there under automatically determines whether a measurement makes sense or not. Ear-Phone also implements the so-called calibration in situ, which means a simple calibration which can be performed by only the general public. Huge disadvantage of this system is its closeness and inaccessibility.

- Bike Net [14] - An experimental mobile sensor system designed for cyclists. It uses smart phones equipped with GPS, which are connected to additional external sensors. The system records the traveled distance, speed, burned calories, terrain roughness, $\mathrm{CO}_{2}$ levels and noise levels in the area. This data could be stored on a simple web portal called Bike View [15], which allowed to visualize routes and sensor measurements on the map. Currently the application is out of operation.

- Noise Tube [16] - This is currently the most advanced open and accessible application. This project was initiated in 2004 in a Sony scientific laboratory in Paris [17]. Currently, this project continues in the laboratory of computer languages at the Vrije Universiteit in Brussels. This application has three main functions: Measuring the noise level, locate it and tag it by hand description (how much is disturbing or what is the source of noise). Data are then wirelessly transmitted to the Noise Tube server. If a user logs itself in to the server, she is able to track their trajectory noise in the web interface of Google Maps. The application is primarily intended for the research of a community work with mobile applications, and community work with environment issues, the mobile application for measuring noise is very high quality. In terms of the measurement of noise and access to data, this app has considerable limitations: 
o It does not allow universal machine processing and accessing to noise data, as the proposed application interface is local and does not reflect the sensory standards and does not allow a broader machine processing of given tags, these characters are treated as a cloud data.

o Noise situation is mapped to the street network on the server, but this mapping does not reflect possible errors in the position measurement.

o Application has options to categorize the different models of mobile phones into the quality classes, but does not evaluate the quality of individual measurements. It does not take into account the spatial or temporal circumstances (such as adjacency of measurement points, time of day, etc.).

\subsection{Character of the Noise Data from Smartphone Crowdsourcing Applications}

The main feature of all these systems here is focus on the sensor platform-a mobile phone, and on the ways in which data may be collected, how the phone can be calibrated and, where appropriate, to eliminate the worst interference when measuring. This approach is logical because without quality and reliable sensor it is not possible to obtain high-quality sensor data. The experience gained from these projects can be summarized as follows:

- Mobile phone platform as the sensor platform is suitable for measuring noise under these conditions and restrictions:

o Mobile phone is a multifunction device; it is necessary to monitor its use.

o Mobile phone must have an Internet connection.

o Calibration of mobile phones is a very sophisticated issue, but automatic calibration is a solvable problem.

o One of the biggest problems is battery life, which is consumed mainly by GNSS sensor and by data transmissions.

- Noise data obtained by measuring using a mobile phone has the following limitations:

o They are incomplete. There must be an algorithm for its adjustment and completion, or at least to determining the degree of completeness.

o They are spatially inaccurate, so must be spatially refined.

o They are focused on serial measuring.

o They must be anonymized.

However, the main feature of such works is the pursuit of technical-engineering view on measuring, which causes a certain distance from other segments of noise data processing. While most of the problems associated with mobile phones' measuring is at least partially solved, given works are only marginally engaged in further processing of the measured data, which primarily means:

- Quantifying and eliminating uncertainties associated with position errors of small and cheap GNSS sensors which are a usual part of today's smartphones,

- Quantification and classification of data in space and time, including in particular. 
o Detection of a noise source based on the closeness.

o Quantifying the impact of noise on the surroundings.

o Detection of spatial and temporal patterns, classification and analysis of such patterns.

- Visualization noise levels and their uncertainties.

o Setting basic rules and procedures, a survey of algorithms.

o Interpretation of measured values by users.

- Openness of systems for further use by other systems and surveying of open standards for the transmission of sensory information, both in the raw and in the processed form.

This last point is particularly important because if the systems declare necessary openness both in the data and in the procedures, it is more than appropriate to exploit the possibilities of open data formats. Although these systems often have their own open application interface, the form of integration between particular noise measurement systems was not sufficiently explored.

\section{Specifics of Noise Measurements Using a Mobile Phone}

The main difference of noise mapping using mobile phones from traditional mapping is the approach to classification of noise sources. Current applications, which are using mobile phones for noise measurements utilize person-centralized approach in which the mobile phone is used as a noise-dose meter, which indicates the overall affection rate by noise for one particular person (an equivalent of radiation dosimeters). Noise level along with time and eventually position is recorded every second (which corresponds to the "slow" measurement standard for standard noise meters).

This approach is, in comparison with conventional mapping of the spatial distribution of noise, considerably easier. There are primarily not distinguished individual sources of noise, including noise, which is created by user. Information about the position of measurement is secondary, may not even be filled, and is used more for orientative purposes. For information purport, such work with a location is quite sufficient, but in case of study of the spatial distribution of noise there should be a somewhat more sensitive approach.

Despite these shortcomings, it can be said that even the dosimetry data, if collected sufficiently accurately, can be used for compiling maps of spatial distribution of noise or even be combined with data from conventional measurements. This approach is also used in a number of mobile applications that allow to visualize the measurements in the map, assuming that measurements are performed while walking.

\subsection{The Issue: Uncertainties of Measurements of Noise by Mobile Phones}

For measuring noise is in practice very difficult to determine the function of 
quantities of individual sources of noise measurement errors. Standards ISO 3745 [18] and ISO 1996-2 [19] identify some of the most important sources of error and their overall contribution can be written as follows:

$$
L_{\text {Aeq,true }}=L_{\text {Aeq, } m}+\delta_{\text {slm }}+\delta_{\text {sou }}+\delta_{\text {met }}+\delta_{\text {loc }}+\delta_{\text {res }}+\varepsilon
$$

where $L_{A e q}$, true is true equivalent sound pressure level adjusted by weighting filter A, $L_{A e q, m}$ sound pressure level measured by noise meter and adjusted by the weighting filter $\mathrm{A}, \delta_{s l m}$ is an error in the measurement chain (sound level meter in the simplest case), $\delta_{s o u}$ is an error caused by the difference from ideal operating conditions of noise source, $\delta_{\text {met }}$ is the error caused by meteorological conditions different from ideal, $\delta_{l o c}$ is a positional sensor error, $\delta_{r e s}$ is an error on residual noise level and $\varepsilon$ residual error. $\delta_{\text {sou }}+\delta_{\text {met }}$ are for static measurements often obtained directly by measuring in the point of interest, but when measuring using mobile phones on the move, only meteorological data available often are, and these only from the remote weather stations.

Uncertainty of each measurement can then be expressed by the error propagation law as:

$$
u^{2}\left(L_{\text {Aeq, }, m}\right)=u_{\text {slm }}^{2}+u_{\text {sou }}^{2}+u_{\text {met }}^{2}+u_{\text {loc }}^{2}+\left(c_{\text {res }} \cdot u_{\text {res }}\right)^{2}+\varepsilon^{2}
$$

where $c_{r e s}$ is a sensitive coefficient for residual coefficient (other sensitive coefficients are equal to 1.0 ).

Table 1 of ISO 1996-2 [18] contains an overview of the measurement uncertainty for the A-equivalent noise level. Higher uncertainties are to be expected on maximum levels, frequency band levels and levels of tonal components in noise.

The article focuses primarily on uncertainty of localization, but preliminary is necessary for clarity, at least briefly discuss the accuracy of the actual measured noise level in the case of mobile phone measurement.

\begin{tabular}{|c|c|c|c|}
\hline \multirow[b]{2}{*}{ Criterion } & \multicolumn{3}{|c|}{ Algorithm } \\
\hline & Raster & Simple vector & $\begin{array}{l}\text { Vector with } \\
\text { metadata }\end{array}$ \\
\hline Computational complexity & Linear & Logarithmic & Logarithmic \\
\hline $\begin{array}{l}\text { Checking record } \\
\text { correct location }\end{array}$ & No & Yes & Yes \\
\hline $\begin{array}{c}\text { Capturing more } \\
\text { complex noise situations }\end{array}$ & No & Partially & Yes \\
\hline $\begin{array}{c}\text { Dependence of position } \\
\text { inaccuracies on the cell size }\end{array}$ & $\begin{array}{c}\text { In both } \\
\text { dimensions }\end{array}$ & $\begin{array}{c}\text { In one } \\
\text { dimension/No }\end{array}$ & $\begin{array}{c}\text { In one } \\
\text { dimension/No }\end{array}$ \\
\hline $\begin{array}{l}\text { Data size growth while } \\
\text { increasing accuracy }\end{array}$ & Quadratic & Linear & Linear \\
\hline
\end{tabular}

Table 1. Comparison of methods for determining spatial measurement noise uncertainty. 


\subsection{Accuracy of Mobile Phones' Microphones}

It is obvious that the accuracy of mobile phone sensors (microphones) is lower than the accuracy of certified noise meters. This drawback, however, is possible to almost completely eliminate by using accurate calibration, at least in case of noise measurements of surface transport.

The laboratory experiments have shown that sensors built in mobile phones have a surprisingly high accuracy when measuring the acoustic noise levels, which is, while applying correct calibration and measurement interval 1 second, in the range of about $35-110 \mathrm{~dB}$ gets below the value of $\pm 1 \mathrm{~dB}$ [20] [21].

Field tests in synergy measurement with the noise meter of Class 2 according to IEC 61672-1 [22] shown that during normal measurement of urban noise situation also deviation of $\pm 1 \mathrm{~dB}$ from the values measured by given noise meter [20] [21]. For the actual level meters of Class 2 was detected uncertainty of \pm 2 $\mathrm{dB}$ [23]. For accurate practical results preliminary synergistic measurement with certified Class I noise meter was performed wherein deviation does not exceed $\pm 2 \mathrm{~dB}$ in any of the cases of non-impulse noise.

The disadvantage of microphones from mobile phones is mainly a lower dynamic of sensor and the resulting slower response, therefore these sensors are not suitable for measurement of impulsive noise, but for measuring of most cases of noise from ground traffic and many kinds of neighborhood noise are sufficient.

Uncertainty of microphones precision varies from type by type and also piece by piece. Issues of calibration of individual phones are quite extensive and are outside of this text scope. Basic information can be obtained e.g. in [24].

\subsection{Positional Accuracy When Measuring Noise}

Mobile phones, especially in urban areas, suffer from higher inaccuracies in positioning. This is essentially a problem of GPS device, which may receive reflected radio signal instead of direct, thereby calculate erroneous distance between GPS transmitter and receiver, which consequently leads to erroneous positioning. The inaccuracy may be in the order of first units to tens of meters. In some case the location of the resulting data points is automatically adjust5d, for instance, when measured on the street network, but the location data will indicate the location inside buildings. Such results, however, have limited relevance.

A significant problem in the interpretation of noise data measured by mobile phones is therefore spatial accuracy (localization) of the sensor. While for certified measurements is the position of the sound meter precisely known and often is standardized so that individual measurements are comparable (e.g. $2 \mathrm{~m}$ from the center of the traffic stream, 4 meters above ground level, min. $2 \mathrm{~m}$ from the facade of the house, etc.), for short measurements using mobile phones is just very difficult to observe all the prescribed parameters. Accuracy of the mounted GPS receivers, which are often reduced the urban environment also plays a very 
important role. As the practice shows accuracy of GPS (and assisted GPS) receivers in mobile phones varies widely and common deviations reach values from 3 to $10 \mathrm{~m}$, and in some cases even to $40 \mathrm{~m}$ [25].

In the case when an accurate knowledge of the position of a noise sensor is not guaranteed (at least in the order of decimeters), it is not possible to determine noise value at the place of its origin and thus obtain information about the noise situation in the area. Values are only valid for the place of measurement, which is itself known with considerable uncertainty.

Maximum uncertainty of noise level in $\mathrm{dB}$ increases with uncertainty of the distance, in case of a point source of noise radiating in all directions, according to noise level equation:

$$
L=10 \log \left(P /\left(4 \pi\left|r^{2}-r_{0}^{2}\right|\right)\right)
$$

where $P$ stands for power of sound waves at the source, $r$ is the actual distance from measurement point to the noise source and $r_{0}$ represents indicated (e.g. measured using GNSS) distance from measurement point to the noise source. If the noise source is a line, is in case of assignment of measurement point to the correct line segment, where is the impact from another segment of the line leading in different direction negligible, maximum uncertainty of the noise level can be obtained in $\mathrm{dB}$ as:

$$
L=10 \log \left(P /\left(2 \pi\left|r-r_{0}\right|\right)\right)
$$

Because we do not know power of sound waves at the source, to determine the uncertainty of the level of noise at a certain probability level is necessary to determine the most probable deviation from the measured noise level. The highest positive value of uncertainty may be, for a point source, determined by the equation:

$$
+u_{L}=L_{r}-L_{s \max }
$$

where $L_{r}$ is the measured noise level at a distance $\mathrm{r}$ from the noise source, $L_{\text {smax }}$ represents noise level at a distance $r$ from the source of the noise, but if the measured noise level $L_{r}$ would apply to point at the distance $r+e$, where $e$ is the uncertainty value of position in given length rate (e.g. in meters), therefore, for the point furthest from the noise source at given confidence level.

The highest negative value of uncertainty according to equation:

$$
-u_{L}=L_{r}-L_{\text {smin }}
$$

where $L_{r}$ represents the measured noise level at a distance $\mathrm{r}$ from the noise source, $L_{\text {smin }}$ represents noise level at a distance $r$ from the source of the noise, but if the measured noise level $L_{r}$ would apply to point at a distance e, where e is the uncertainty of value position in the given length rate, i.e. for the point closest to the source of the noise on a given confidence level.

For the linear noise source equations will be analogical. 
Spatial error of $5 \mathrm{~m}$ from a point source of noise that may indicate faulty assignment noise level to even $15 \mathrm{~dB}$, from a line source (which is for example a road with regular traffic) to about +6 and $-10 \mathrm{~dB}$.

Problem grows even more, if users measure the noise when walking, which is currently among the most anticipated applications of crowdsourcing method of measurement. Some of the services used (e.g. Noise Tube) are solving these inaccuracies by assign recorded noise value points to the appropriate points on the street nearest from the recorded position [26]. Such an assignment itself is insufficient, there should still retain significant positional deviations. It is also appropriate to supplement recorded values user data (user himself specifies the relative position, such as street name, closest house number and the estimate of distance from it, etc.).

The error in this field can then grow even more if insufficiently rigorous procedures and tools to evaluate the noise are utilized. The most common procedure for the aggregation of measured values is a regular square grid. Due to GNSS receiver inaccuracies, the most appropriate size of the edges of the square grid cell is considered to be about $20 \mathrm{~m}$ so that the value with the highest probability will be actually incorporated into the correct square. But for the calculation of noise in the vicinity of the noise source is required spacing between the calculation points up to $10 \mathrm{~m}$ [27]. Therefore, the position of the individual measurements must be adjusted.

\subsection{Operating Conditions of Noise Source}

Operating conditions of noise sources often varies throughout the day. For example, when measuring the noise on the road network, it is necessary to take into account the current time due to rush hours, all in terms of the day and the week, month and year. Depending on these conditions, a variety of noise descriptors that describe the situation was introduced. Among the most commonly used descriptors include:

- Sound pressure level $(L)[\mathrm{dB}]$ — may be weighted (e.g. by using the weighting filter A or C).

- Statistical noise level $\left(L_{\mathrm{n}}\right)$-noise level exceeded for $\mathrm{n}$ percent of the measured interval. (Indicators $L_{1}, L_{10}, L_{50}, L_{90}$ and $L_{99}$ are used for a rough estimate of the maximum noise levels, noise, median and background noise.)

- The equivalent continuous noise level $\left(L_{\mathrm{eq}, \mathrm{TL}}\right)$ - Theoretical noise level, which describes the noise that varies considerably during the timed interval. If the input value is weighted according to the acoustic weighting filter $\mathrm{A}, L_{\mathrm{Aeq}}$, T.

- The level $L_{\mathrm{den}}$ (day-evening-night) —an indicator of the overall noise.

- Level $L_{\text {day }}$ (day)-indicator for interference-day period, defined as the weighted (by using the weighting filter A) long-term average sound level according to ISO 1996-2 [19] designed for any day of the year.

- Level $L_{\text {evening }}$ (evening) - an indicator for interference evening period, defined 
as the weighted long-term average sound level, fixed for all the evening periods of the year.

- The level $L_{\text {night }}$ (night) —an indicator for disturbances during sleep, defined as the longer-weighted average sound level, set for any night of the year.

From the perspective of crowdsourcing noise measurement is usually sufficient just to record the precise time of measurement, in order to correctly assign given records. The actual uncertainties can then be calculated according to evaluated descriptor.

A much larger problem is the insufficient length of measurements. None of the above mentioned systems does not answer the question whether processed data are sufficiently representative for an objective assessment of noise levels at the site. The criterion of representativeness deals to the character of the behavior of the noise source.

\subsection{Weather Influence onto Measurements}

Quite a considerable influence on the measured noise level has the weather. There are issues on precipitation (rain drumming up around the sensor), on thunder and especially on the wind, which can greatly affect measured values. Professional sound level meters often use so-called wind guard and, when outdoor, also temperature, humidity and wind speed are measured in addition to the noise level.

In the case of mobile phones, compliance with such requirements is relatively complicated, so it is necessary to rely on the expertise of users, and also increase the potential uncertainty of measurement in the case of higher wind speeds or gusts recorded in the nearest available meteorological stations, then might be necessary to excrete such a wide range of measurements, which limits noise measurement capabilities, especially in variable weather. Also there is a possibility of noise frequency analysis that could eventually detect wind gusts.

\subsection{Influence of Residual Noise}

Residual noise during measurement using mobile phones represents mainly a noise that is emitting by the user alone. It can be both noise caused by walking or biking, and above all the noise from the speaking or operating the phone. In the case that we tolerate the user to move during measuring, there should be no other option than to accept the fact that we can then evaluate the noise up from a certain level, which is about $10 \mathrm{~dB}$ higher than the noise caused by walking or driving. Noise level of walk with soft soles on hard subsoil reaches (from our experience) values $30-40 \mathrm{~dB}$, in the case of heels or walking on gravel may reach up to $70 \mathrm{~dB}$. It is therefore necessary to exclude or mark such measurements properly [28].

In case of speaking we do not have in mind talking of user itself (it is obvious that user can't simultaneously talk and measure noise), but rather speaking of people around. Especially when we measure noise levels from road traffic, but 
the occasional conversation of pedestrians around should significantly disrupt collected data. In this case it is appropriate either interrupt measurement or rely on the frequency analysis, which allows to detect human conversation and then the measurement is automatically discarded. If the data on the frequency distribution are not transmitted to the post processing, it is necessary to carry out these detections and adjustments in the mobile phone, which is very computationally expensive [29] [30] and is typically not performed.

\subsection{Summary}

There are numerous negative impacts on noise measurements using a mobile phone. Aside from incorrect device operation, it is mainly the effect of measurement chain: quality of microphone calibration, processing of noise signal and influence of residual noise from other sources. These areas are dependent upon the input settings or direct measurement circumstances. Their modification on subsequent processing is very problematic. Quantifying of uncertainty on these effects is directly dependent on knowledge of the whole system.

Additional uncertainties arise from the spatial accuracy, from weather situation and from operating conditions of the noise source. In crowdsourcing, virtually no attention is paid to these uncertainties. But they can be quantified and modified during post-processing. But, on the contrary, during measurement they can be evaluated only by greatly increased effort. Using the following techniques and procedures, it is possible to generate a standardized data set which uncertainties are sufficiently reliably quantified and which can thus enter into the subsequent analysis.

\section{Processing of Positional Errors of Environmental Noise Data from Surface Transport Collected through Mobile Phones}

Position of measurements during crowdsourced measurement of traffic noise is necessary to bind primarily to the noise source. This is, from the perspective of surface transport measurement, a track line (e.g. road, rail). Taking i.e. into account the normal noise level emitted by an average passenger car during continuous ride at speed of $50 \mathrm{~km} / \mathrm{h}$ on normal asphalt surface, when an error $5 \mathrm{~m}$ occurs in the position measuring, the measurement error can reach $6-10 \mathrm{~dB}$. [26] Data obtained with this error are therefore highly approximate and may not be used in essentially any more detailed analysis.

However, the position of the measured data can be evaluated such as to make clear what the position error and the value of its contribution is. Then we can evaluate the uncertainty of measurement in $\mathrm{dB}$ or fix the position (if possible).

\subsection{Aspects of Evaluation of Positional Errors in the Raster Grid}

The simplest and perhaps the fastest way of assessing positional error is to use 
the grid to which both measured values and values of positional uncertainty are aggregated. Data can be clustered into grids based on their recorded position and positional uncertainty both on the basis of estimated spatial error provided by GNSS receiver. However, these data are often not available or the spatial error estimate is unreliable. This applies especially to the urban environment, which is suffering from so-called Street canyon effect, and also in heavier forests where GNSS coverage is usually quite random.

For more reliable determination of uncertainties is thus appropriate to use data, which already have sufficient accuracy-on the one hand for the positioning of the source-and if possible, especially the estimated position or trajectory, on which the user is moving. For evaluation of noise from land transport this today in practice means the use of spatial databases (maps) of the road network. These databases itself may not suffer from too high positional inaccuracies and must be as complete as possible.

\subsection{Algorithm for Evaluation of Positional Errors in the Raster Data Grid with Street Data}

Street data are nowadays usually stored in vector databases. For ease of cluster analysis, it is therefore necessary to convert them into raster grid. Thereafter, on the basis of the location attribute, each value of the noise level of the raster cell is averaged and stored in a square raster. In a similar manner is calculated the average deviation of the position of the measuring points from the noise source (so the street, represented by the raster cell or as a direct line string). The result can be easily visualized and further clustered. Overall scheme is depicted on Figure 1 .

Between the main features of this algorithm belongs:

- High speed of processing and visualization.

- Simplicity of implementation.
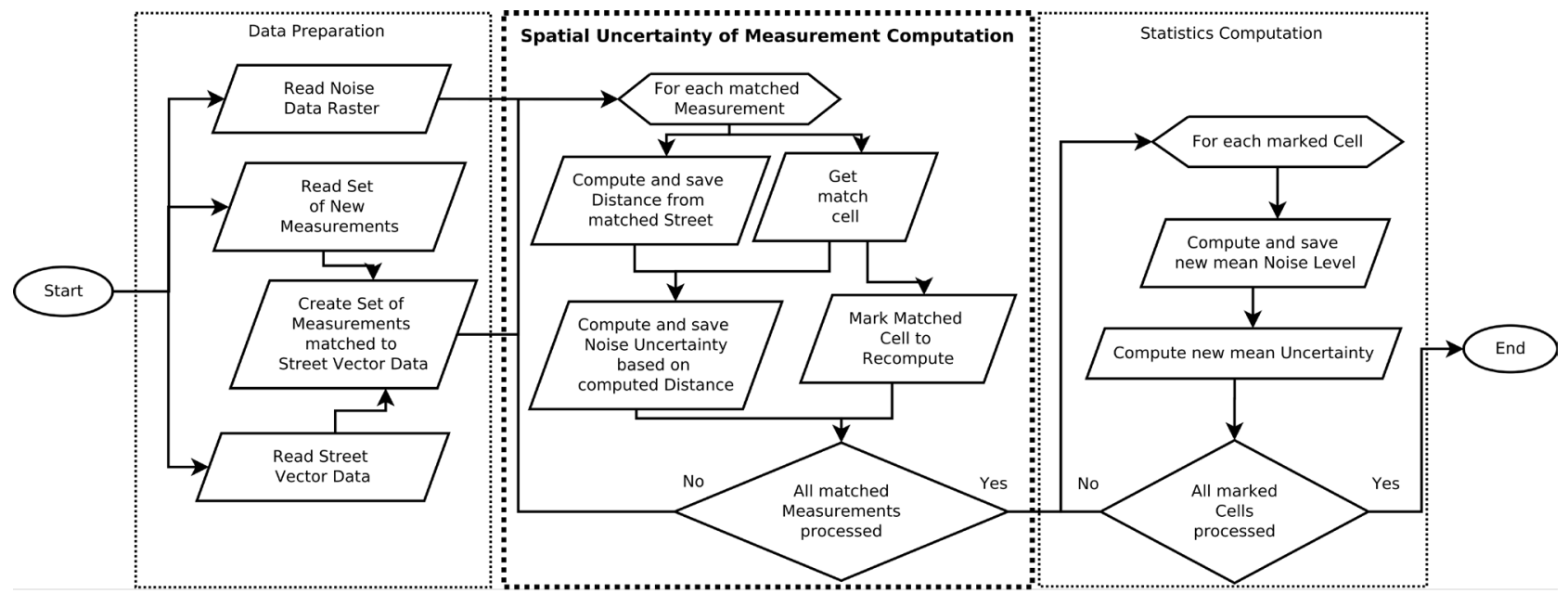

Figure 1. Determining spatial uncertainty for noise measurements using raster data. 
- It can be, due to the shape of the Earth, applied only locally, so it is important to determine the area to which it will apply.

- It can't capture more complex noise situations, whether in horizontal (noise barriers, intersection, multi-lane roads, etc.) and vertical orientation (especially bridges).

- Assigning a value to the noise source is limited by the size of the aggregating cell and is heavily dependent on randomness of its position.

- It is not detected whether values are assigned to the correct cells.

- Accuracy of determined uncertainties as well as transitions of noise levels is highly dependent on cell size. In the case of small cells in a large area is the grow of the volume of needed data and the processing speed inversely proportional to the area of the cell.

Despite these disadvantages, the raster method is used for processing and visualization of noise in almost all projects, including those mentioned in Chaption 1.1. Most of them do not even take into account the street network yet.

It should also be pointed out that, when assessing the noise situation on land transport using square grid, the shape of the road network plays an important role. In the case that streets are mutually perpendicular, a square grid with the cells oriented in the same direction with the street network allows sufficiently precise and unambiguous assignment of points of the road network to the raster. However, if streets diverge, this situation is much more complicated. Theoretically, it is possible to use other types of raster (triangular, hexagonal, see e.g. Noise Map [7]), but work with these types of raster is much more complex and many advantages of the raster approach is thus considerably reduced.

\subsection{Aspects of the Evaluation of Positional Errors in the Vector Model}

Vector model allows to evaluate the positional error in with several orders higher accuracy compared to raster model. This in turn allows to map noise sources' and measurement points' relative position more accurately. The result could ideally serve to recover the value of sound power of noise sources on sufficient precision. At least, these data can then be used to assess the overall noise situation. However, evaluation of noise levels in the vector model requires much more challenging data preparation. Also calculation itself is also much more complex and takes incomparably longer. But, according to the development of computer technology in recent years, these restrictions cease to be serious. Positional error in the vector model can be determined as the distance to the nearest point on the line of the interpolated position along places designated as vertexes (designated by a user, or a specialized algorithm).

\subsection{Simple Algorithm for Evaluation of Positional Errors in Vector Model with Street Data}

Data on street network in vector form are nowadays represented mainly by so- 
called line chains (line strings), which in fact are strings of point coordinates. However, from the perspective of assigning noise levels to the streets, represented by the lines, is assumed that the value of both noise levels and the uncertainty value will be assigned to a specific section of the line, where the noise situation is practically homogeneous.

In order to determine which part of the line noise is homogeneous, there is hardly an alternative to a regular line divided into segments, where the noise situation will be assessed separately. The size of individual calculation segments can be adjusted according to the required accuracy in terms of noise measurements by mobile phones, but can be based on the values specified in chapter 2.3. We can say, that the basic segment length should vary in the values of about 10 $20 \mathrm{~m}$, because shorter segments already does not provide any substantial increase in accuracy. The depiction of this algorithm is on Figure 2.

The algorithm itself is divided into several phases, which take place separately. Firstly, it is necessary to determine the position of each segment in the street network and then is possible to aggregate measured values and their uncertainties. Subsequently aggregation of segments with similar data may occur, which saves data space and time for further processing and visualization. The main features of this algorithm are:

- Larger memory and performance requirements. It is appropriate to process areas sequentially.

- Rather more complex implementation.

- In case of suitably chosen coordinate system, the possibility of a global and seamless processing enables fully automated handling without human intervention.

- Is able to partially capture a more complex noise situation in terms of vertical, but still just with limits (such as bridges) and partly also horizontal complex situations (e.g. noise barriers, intersection). But it can't quite adequately
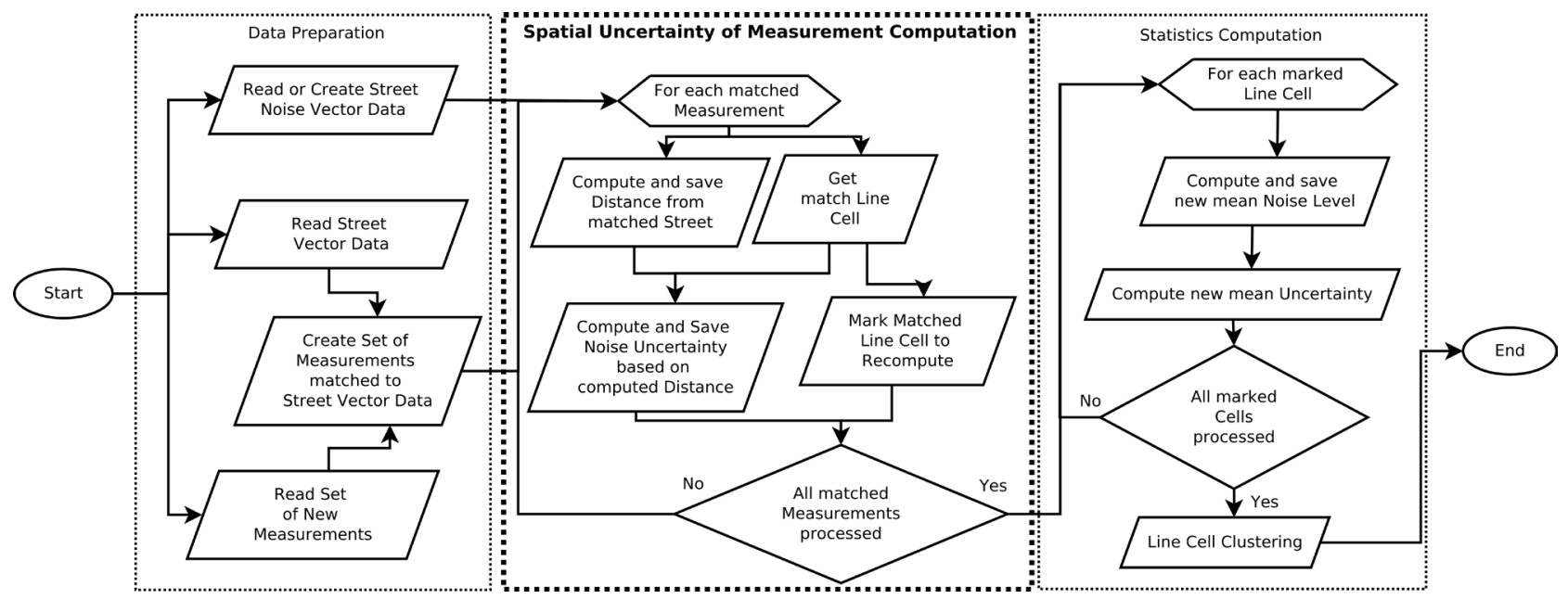

Figure 2. Determining spatial uncertainty for noise measurements using vector data. 
assess the situation in cases of asymmetrical noise situations, more lanes or composition of multiple noise sources (e.g. tramway strip between road lanes).

- Assigning a value to the noise source or noise emission levels on the street is limited by the size of the aggregate cell only in the longwise direction. It is no longer entirely dependent on a random location of cell boundaries.

- There is a check of the correct assignment of measured value to the source, because the values are assigned sequentially based on prior knowledge about the direction of monitoring of a series of measurements.

- Accuracy of perceived uncertainties as well as transitions of levels depends on the cell size and density of the road network, in the case of small cell on a large area is growth in the volume of the necessary data as well as processing speed inversely proportional to the length of a side of the cell.

Noise evaluation using this algorithm also brings the need to address issues which were not necessary to be addressed in the evaluation of noise using raster network, because of its lower spatial accuracy. In case of noise data obtained by pedestrians using mobile phones there is issue on the position of the measurement points on the given street cross-sectional profile. Knowledge of the situation in street's cross section allows accurate determination of uncertainty. For this processing method, it is necessary to obtain additional data from the user.

\subsection{Algorithm for Evaluation of Positional Errors in the Vector Model with Street Data and Data about the Relative Position and User Movement}

As should be obvious from everyday experience for all of us, there are a huge variety of street cross-sections, but most vector street network databases represent the street only as a simple line. But if the data about the width of the streets or sidewalks position is available, is possible to determine the distance from the source of pedestrian traffic noise more precisely. And even in the event that such information is not available, it is advisable to remember the information on which side of the street the user is measuring, because from these data is possible to detect any asymmetry of the noise situation later, which may substantially refine the further processing and interpretation of noise data. The algorithm scheme is on Figure 3.

For proper function of this algorithm is primarily needed to determine the relative position of the user. One of possible ways may be an extension of the data that a user enters during measurement. In the case that user enters, on what side of the street he/she is (from the perspective of the direction of the walk), it is possible to assign the position of a point not directly to the street, but for example to the sidewalk (if data about sidewalks are available), or at least the average distance from the center street (e.g. by knowing the number of lanes, building layouts etc.).The main features of this algorithm are: 


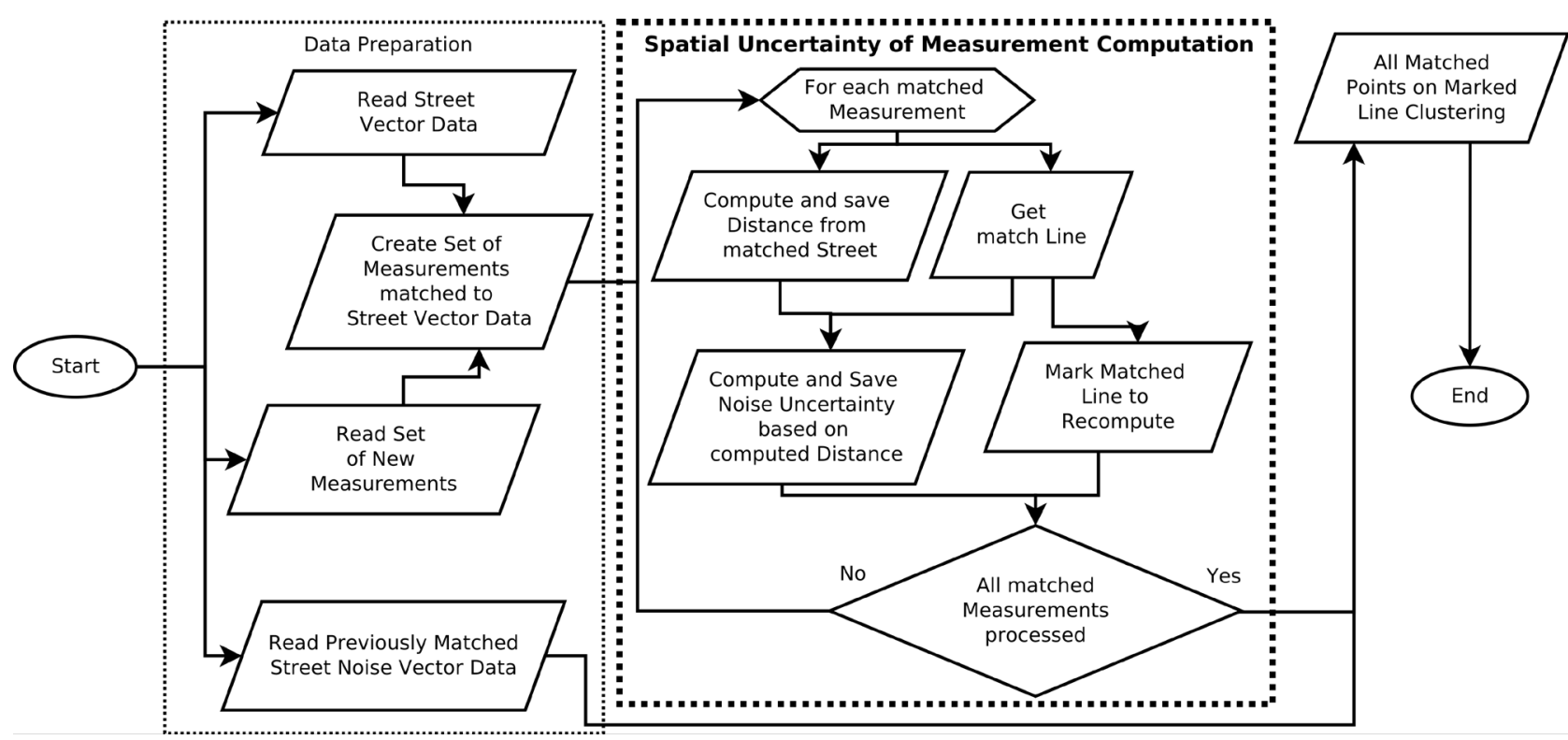

Figure 3. Determining spatial uncertainty for noise measurements using vector data and point clustering.

- Large memory and performance requirements, it is appropriate to process areas sequentially.

- It is arduous for input data, both in terms of individual volunteers as well as the underlying data about street network.

- Challenging implementation.

- In case of suitably chosen coordinate system there is a possibility of a global and seamless processing, which enables fully automated handling without human interventions.

- Is able to capture a more complex noise situation in terms of both vertical (such as bridges) and the horizontal (noise barriers, intersections), including asymmetrical noise situation, more lanes or composition of multiple noise sources (e.g. tramway strip between road lanes).

- Assigning a value to the noise source or noise emission levels on the street is limited by the size of the aggregate cell only in the longwise direction. It is no longer entirely dependent on a random location of cell boundaries.

- There is a check of the correct assignment of measured value to the source, because the values are assigned sequentially based on prior knowledge about the direction of monitoring of a series of measurements and on the basis of additional data from the sensor.

- Accuracy of perceived uncertainties as well as transitions of levels depends on the cell size and density of the road network, in the case of small cell on a large area is growth in the volume of the necessary data as well as processing speed is inversely proportional to twice the value of the length sides of the cell.

The use of this algorithm allows, when quality of input data is sufficient, not just quite reliably evaluate the positional uncertainty, but also largely eliminate 
this uncertainty. Implementation and testing of this algorithm has been performed in the analytical module of the Mobile Noise system (see chap. 5.1).

\subsection{Mapping User's Position on a Street Network}

The processing of inaccurate location data on street network is also related the problem of assigning the correct position of the waypoint into the street network (onto the correct segment of street). In the case of low or moderate buildings and common street network appears adequate use of some of the classical topological algorithms (see e.g. [31]), because spatial error is not particularly significant due to the density of conventional road network. In case of a much finer footpaths mesh, it is necessary to choose a more precise approach.

Because that from the mobile phone should not be available data about actual walking variables (it is possible to obtain data about the direction of movement and predicted circular deviation at best), it is not possible to use one of the "map-matching" algorithms for instantaneous positioning. Therefore, an alternative algorithm that exploits similarities in the general azimuth and turning points of user's way and footpaths was assembled and tested:

The work of this algorithm in a normal situation proceeds similar to the topological algorithm. In case of exceeding the limit of the distance measured from a point to the line or in case of some topological ambiguities in determining the position is certain number of previous and subsequent measurement points (depending on the size of the error) generalized by a simplification algorithm (e.g. Douglas-Peucker [32] or Visvalingam [33]). Thereafter individual azimuths of generalized sections are compared with azimuths of the next segment of streets and sidewalks.

\subsection{Use of Clustering in Noise Data Post-Processing}

When using the cell model in the vector data, only uncertainty in the perpendicular direction to the street is evaluated. Classification of the measurement point to a particular segment can be controlled only on the basis of the sequence of individual points in time and the user movement along the line. Boundaries of individual segments with homogenous noise situations are determined on the basis of predetermined criteria. Clustering of individual segments into the acoustically homogeneous units is based on the similarity of the noise levels in individual adjacent segments.

However, individual measuring points can be classified directly on the street and segments with homogenous noise situations produced using clustering of all points along the road. This leads to elimination of the arbitrary component for determining cell size. Individual sizes of homogeneous segments can thus be determined directly from the data. The disadvantage of this algorithm is, when updating the additional data, a necessity to analyze all data again, as moving of existing segments' boundaries is very difficult. 


\subsection{Summary-Comparison of Individual Methods for Determining Spatial Measurement Uncertainty of Traffic Noise Using Mobile Phone}

Based on the above text it can be summarized the advantages and disadvantages of different algorithms to Table 1.

Among the main advantages of vector algorithms belongs mainly their higher accuracy at a lower volume of data required for processing. In case of lower accuracy is calculation using grid faster and requires less memory, in case of increasing the resolution of data, however, increases according to area by the second square. In the case of increasing data is vector algorithms dependent primarily on the details of the underlying geographic data about street network and with higher accuracy requirement increases linearly (if side street on which the user is located is used, the values are doubled), if data are clustered after assigning the street network, a value is constant and is equal to twice the number of processed points.

Thus, the advantages of vector processing take effect there, where a necessity for further data use exists and their simple visual interpretation is not enough.

\section{Uncertainty of Measured Noise Level Caused by Traffic Fluctuations}

One of the basic issues of noise measurement using mobile phones stands for how long is necessary to measure to ensure that the resulting value is representative. Within a single measurement of noise from road traffic, it is possible to calculate the uncertainty of measured value according to time from the number of passing vehicles from the equation:

$$
u_{\text {sou }}=\frac{C}{\sqrt{n}}
$$

where $n$ is the number of passages and $C$ coefficient type of transport. In the case of mixed traffic, it is possible to determine $C=10$, whereas for only light passenger cars $C=2.5$, and for only heavy trucks $C=5$. On the less busy streets, where it is possible to record each car passage separately, may be the uncertainty calculated automatically after measuring end. The user should also be able to select a type of traffic, if she knows that along the road is moving only certain type of vehicle.

Some methodologies, i.e. [34] [35] recommend calculating the minimum measurement length, which could be considered as representative. For this approach is of course necessary to know at least the number of vehicles, which passed given measurement point per hour. If this value is higher than 100 , following equation can be used:

$$
t_{\min }=\left(\frac{4000}{q}+\frac{120}{r}\right)
$$


where $q$ is the number of vehicles (trains) passing through the measured point per hour and $r$ the number of samples per minute. Result is in minutes.

If the user knows the number of passing cars (e.g. he counts them during the measurement), it is appropriate that this value is entered after the measurement of noise for each road segment metadata. In this case, it is possible to specify (shorten) the minimum period of non-collaborative measurements, after which it is possible to take results into account and display them on the map. In other cases, it is recommended to perform non-collaborative measurements for unchanged conditions for at least 1 hour aggregated.

If the user does not track or are not able to enter the number of passing vehicles, it is possible to estimate the uncertainty of transport irregularity due to the kind of communication. The type of traffic situation must be taken into account (given day, week and year time, e.g. whereas if it is morning or afternoon peak, Saturday, or middle of the working week, etc.).

For a very rough estimate of uncertainty there can be also used following equation (for streets with the passage of more than 100 vehicles per hour):

$$
u_{\text {sou }}=\sqrt{60 / t_{\min }}
$$

where $t_{\min }$ is the sum of durations of all measurements in minutes.

Given that both the standard level meters, as well as applications for mobile phones are using the "fast" method of measuring, where value is read every second, is sufficient for determining the length of measurement for each noise homogeneous segment just to sum all the number of measurements assigned to it and divide the resulting value by 60 .

If less vehicles, than about 60 per hour, passes the road, road is losing noise line source character and local factors will have a predominant influence onto noise pollution levels with high certainty. Instead of the sound pressure level is thus more appropriate to describe the noise situation in other indicators, such as the number of exceeds of the specified noise level for a given unit of time. Applications processing noise data should be able to identify these factors and adequately work with them (e.g. calculate alternative indicators).

It should be noted that noise descriptors described in chap. 2.4. have clear definitions of the time during the day, week and year, and therefore it is necessary to assign measurements, which took place at different times, to right values and not mix them with other types.

\section{Implementation and Testing of Algorithms for Processing the Noise from Road Traffic Measured by Mobile Phones}

Above mentioned algorithms were implemented and tested as part of a prototype system for monitoring, evaluation and fusing data on noise levels called Noise Mobile. 


\subsection{The Mobile Noise System}

This system is designed to process data measured using both mobile phones and using other types of sensors. It was developed between 2014 and 2016 at Masaryk University in Brno in the Czech Republic. This system was specifically designed to enable better use of noise data measured using mobile phones and to integrate common standards for sending, storing and querying sensor data.

The system consists of four parts:

- Application for measuring data using mobile phones. Given that there are already several reliable applications for noise measurement using mobile phones, which are open source, and which can be modified and adapted, own application has not been developed. Instead, an Android application Noise Tube was adapted to meet the communications requirements and to provide additional data (see chap. 3.5.). The application is written in Java and data are sent in JSON format and comply with ISO standard Observation and Measurements [36].

- Module for storing and providing data to a third party. This module uses the Sensor Observation Service standardized format [37] for accessing sensor data, both raw measurement data and the modified data. This module is written in Java servlet to serve as Apache Tomcat, as the database is used PostgreSQL with PostGIS extension on a machine with Microsoft Windows 7.

- Analysis module for processing noise data. This module has the task to classify the measured data and perform fusing and data interpretation. This module is written in $\mathrm{C}++$ and uses PostgreSQL database with the PostGIS extension for speed purposes. The module can run on Linux and Windows.

- Visualization module whose purpose is to allow the user to visually interpret the output noise data in a spatial context. The output is made through a web interface that shows raster tiles with spatial information.

- More information and a detailed description of the system can be found in [38].

The actual algorithms are implemented in the analytical model, in the form of so-called. Classifiers. Classifiers are expanding building blocks, which performs data manipulation and analysis.

Currently, classifiers can be divided into three groups:

- Basic classifiers: These classifiers are designed to perform basic analysis and classification tasks of a noise situation, such as aggregating data, calculating averages and uncertainties on the lines etc. They are an integral part of the application. These classifications can be expanded by writing new classes derived from the base class and satisfying given application interfaces.

- Library classifiers: These classifiers have similar aim as basic classifier, but are user defined as a dynamic library with prescribed interface in $\mathrm{C}$ or $\mathrm{C}++$ or callable from $\mathrm{C}++$ and connected before or during a run. These classifiers are 
currently available just for Windows (as dynamically linked libraries, or "dll") and Linux (as shared object libraries, or "so") platforms.

- Database classifiers: These classifiers are text based file in PL/SQL language, which is loaded by a basic classifier with the PL/SQL text file input.

Individual classifiers can also be orchestrated. Configuration of individual classifiers and their orchestration is possible using a configuration file in JSON format, or from the command line.

\subsection{Algorithms Testing and Validation}

All four of the above mentioned algorithms has been tested using data obtained during the measurement campaign between 2014 and 2016. The largest and most comprehensive measurements were made in Jundrov district of Brno in the fall of 2014. Figurants for a few weeks moved only after well-defined routes and use two "calibrated" HTC One phones with customized NoiseTube mobile application. From this campaign a total of 20263 samples is available, the average lateral deviation from the known position of the walkway is $2.42 \mathrm{~m}$ and a standard deviation $2.76 \mathrm{~m}$, which indicates a large variability in measurement accuracy. This variability is due to the type of buildings (in Jundrov mainly low buildings, but also relatively narrow streets) and vegetation cover (grown deciduous trees in places). Determination of the average statistics of error for the entire file is therefore unnecessary, because the measurement conditions are different in different parts of measured locality.

For this reason, have been selected to demonstrate the algorithm three cases:

- A sequence of runs multiple measurements on the street enclosed on both sides of the end walls one-story townhouses. Street is one-way traffic.

- A sequence of runs multiple measurements on the street partially shaded by tall trees, with good quality GPS signal. Street is one-way traffic.

- A sequence of one measuring run on the street heavily shaded by tall trees on one side and a two-story row houses on the other side. The street is a both way traffic.

While the relatively conflict-free case 2 the use of algorithms offers only relatively small improvement in accuracy in the order of meters and the first units of decibels, in case 1, which is typical for older urban environments, the uncertainty results are above the threshold of ISO operational errors $( \pm 3 \mathrm{~dB})$. However, most striking benefits from advanced algorithm for refining the position are reflected in an extreme case 3, which is common in many cities. Due to position error variability it would be necessary to exclude completely all the measurements, which are outlying in direction of segments joining on both ends of right way, especially if they have been inside difficult street network. In this case it is of course possible to assign given measurements to the correct sidewalk (on the basis of topological map-matching with nodes specified by the user) and to determine the possible lateral error. 


\section{Conclusions}

The outputs from the noise measurement using the sensor network consisting of mobile phones, which are used by users for the measurement of during moving for their own objectives, is necessary, for the possibility of the proper interpretation, process further:

It is necessary to evaluate the uncertainty of individual measurements, not only based on errors of the sensor itself, but also on positional, errors from the irregularities in the operation of the noise source, and also on the basis of other errors (e.g. correction on weather, correction to other noise sources which we do not want to track, etc.).

In the case of a well calibrated microphone and a sufficiently high number of measurements is possible to improve the positional accuracy to the accuracy level of geographic base data and also improve the error from irregular operation of noise sources to determine at least approximately the number of passing vehicles from noise peak detection.

It is possible to adjust the error values from the position according to userspecified (or specialized algorithm specified) vertex points. That way is possible to obtain data suitable for further processing: thematic visualization and calculations.

Processing of this data is very challenging for algorithm development, computing power and data transfer. The end users must comply with a large number of principles, otherwise the measurements are essentially worthless.

However, if the processing of noise data is sufficiently rigorous, it is possible to obtain relatively accurate data for a relatively low cost. These data can show similar accuracy as computational data from traffic. So it is possible to perform noise measurements without the assistance of a large number of experts and precise, expensive equipment even in places that are not yet, in terms of noise and traffic infrastructure, too much processed.

\section{References}

[1] Maisonneuve, N., Stevens, M., Niessen, M.E. and Steels, L. (2009) NoiseTube: Measuring and Mapping Noise Pollution with Mobile Phones. 4 th International Symposium on Information Technologies in Environmental Engineering, Thessaloniki, 28-29 May 2009, 215-228.

[2] Kanjo, E. (2010) NoiseSPY: A Real-Time Mobile Phone Platform for Urban Noise Monitoring and Mapping. Mobile Networks and Applications, 15, 562-574. https://doi.org/10.1007/s11036-009-0217-y

[3] Santini, S., Ostermaier, B. and Vitaletti, A. (2008) First Experiences Using Wireless Sensor Networks for Noise Pollution Monitoring. 3rd ACM Workshop on RealWorld Wireless Sensor Networks, Glasgow, 1 April 2008, 61-65. https://doi.org/10.1145/1435473.1435490

[4] Foerster, T., Jirka, S., Stasch, C., Pross, B., Everding, T., Bröring, A. and Jürrens, E.H. (2010) Integrating Human Observations and Sensor Observations-The Example of a Noise Mapping Community. Proceedings Workshop Future Internet 
Symposium, Berlin, 20-22 September2010, 4 p. http://www.arne-broering.de/FIS DE final.pdf

[5] Foerster, T., Díaz, L. and Bröring, A. (2011) Integrating Volunteered Human Sensor Data into Crowd-Sourced Platforms: A Use Case on Noise Pollution Monitoring and Open Street Map. 25th EnviroInfo Conference on Environmental Informatics, Ispra, 5-7 October 2011, 505-510.

[6] Ester, M., Kriegel, H.P., Sander, J. and Xu, X. (1996) A Density-Based Algorithm for Discovering Clusters in Large Spatial Databases with Noise. Kdd, 96, 226-231.

[7] Schweizer, I. (2012) Noisemap: Multi-Tier Incentive Mechanisms for Participative Urban Sensing. 3rd International Workshop on Sensing Applications on Mobile Phones, Toronto, 6-9 November 2012, 1-5. https://doi.org/10.1145/2389148.2389157

[8] Telecooperation (2016) Da_Sense. http://www.da-sense.de/? lang=enUS

[9] HabitatMap (2016) Lunar Logic. AirCasting. http://aircasting.org

[10] HabitatMap (2016) HabitatMap. http://habitatmap.org/about us

[11] Everyavare Project Partners (2016) EveryAware: Enhance Environmental Awareness through Social Information Technologies: EU-Funded Research Project (FP7). http://cs.everyaware.eu/event/widenoise/about

[12] WideTag (2009) WideNoise. https://web.archive.org/web/20160521000951/ http://www.widetag.com/widenoise/

[13] Rana, R.K., Chou, C.T., Kanhere, S.S., Bulusu, N. and Hu, W. (2010) Ear-Phone: An End-to-End Participatory Urban Noise Mapping System. 9th ACM/IEEE International Conference on Information Processing in Sensor Networks, Stockholm, 1215 April 2010, 105-116. https://doi.org/10.1145/1791212.1791226

[14] Eisenman, S.B., Miluzzo, E., Lane, N.D., Peterson, R.A., Ahn, G. and Campbell, A.T. (2009) BikeNet: A Mobile Sensing System for Cyclist Experience Mapping. ACM Transactions on Sensor Networks, 6, 1-39. https://doi.org/10.1145/1653760.1653766

[15] Eisenman, S.B., Miluzzo, E., Lane, N.D., Peterson, R.A., Ahn, G. and Campbell, A.T. (2009) BikeNet and BikeView. https://web.archive.org/web/20120308013134/ http://bikenet.cs.dartmouth.edu

[16] D'Hondt, E., Stevens, M. and Jacobs, A. (2013) Participatory Noise Mapping Works! An Evaluation of Participatory Sensing as an Alternative to Standard Techniques for Environmental Monitoring. Pervasive and Mobile Computing, 5, 681694. https://doi.org/10.1016/j.pmcj.2012.09.002

[17] Sony CSL (2016) Sony Computer Science Laboratory Paris. http://www.csl.sony.fr/

[18] International Organization for Standardization (2012) ISO 3745:2012: AcousticsDetermination of Sound Power Levels and Sound Energy Levels of Noise Sources Using Sound Pressure-Precision Methods for Anechoic Rooms and Hemi-Anechoic Rooms.

[19] International Organization for Standardization (2007) ISO 1996-2: 2007: Acoustics-Description, Measurement and Assessment of Environmental Noise-Part 2: Determination of Environmental Noise Levels.

[20] International Electrotechnical Comission (2013) IEC 61672-1: 2013 Electroacoustics-Sound Level Meters-Part 1: Specifications.

[21] Roberts, B., Kardous, C. and Neitzel, R. (2016) Improving the Accuracy of Smart 
Devices to Measure Noise Exposure. Journal of Occupational and Environmental Hygiene, 13, 840-846.

http://www.tandfonline.com/doi/full/10.1080/15459624.2016.1183014

https://doi.org/10.1080/15459624.2016.1183014

[22] Murphy, E., King, E.A. and Rahusen, S. (2015) Exploring the Accuracy of Smartphone Applications for Measuring Environmental Noise. 44th International Congress on Noise Control Engineering, San Francisco, 9-12 August 2015.

http://researchrepository.ucd.ie/bitstream/handle/10197/7042/Internoise2015 Pape r.pdf? sequence $=4$

[23] Douglas, M. and Erik, A. (2005) Uncertainties in Environmental Noise Assessments-ISO 1996, Effects of Instrument Class and Residual Sound. Proceedings Forum Acousticum, Budapest, 29 August-2 September 2005, 1263-1268.

https://web.archive.org/web/20151010215431/http://bksv.dk/doc/bn0151.pdf

[24] Stevens, M. (2012) Community Memories for Sustainable Societies-The Case of Environmental Noise. PhD Dissertation, Vrije Universiteit, Brussel.

[25] Modsching, M., Kramer, R. and Hagen, K. (2006) Field Trial on GPS Accuracy in a Medium Size City: The Influence of Built-Up. 3rd Workshop on Positioning Navigation and Communication, Hannover, 16 March 2006, 209-218. http://wpnc.net/fileadmin/WPNC06/Proceedings/30 Field trial on GPS Accuracy in a medium size city The influence of builtup.pdf

[26] Duda, P. (2014) Generating Cartographic Representations of Volunteered Environmental Noise Data from Mobile Phones. In: Brus, J., Vondrakova, A. and Vozenilek, V., Eds., Modern Trends in Cartography, Springer, Berlin, 369-383.

[27] Merchana, C.I. and Diaz-Balteirob, L. (2013) Noise pollution Mapping Approach and Accuracy on Landscape Scales. Science of the Total Environment, 449, 115-125. https://doi.org/10.1016/j.scitotenv.2013.01.063

[28] State of New South Wales, Environment Protection Authority (2013) Noise Guide for Local Government. Part 5 Appendixes Washington. Page 5.3. http://www.epa.nsw.gov.au/resources/noise/130127NGLGPart5.pdf

[29] Chu, S., Narayanan, S. and Jay Kuo, C.C. (2009) Environmental Sound Recognition with Time-Frequency Audio Features. IEEE Transactions on Audio Speech and Language Processing, 6, 1142-1158. https://doi.org/10.1109/TASL.2009.2017438

[30] Pfeiffer, S., Fischer, S. and Effelsberg, W. (1996) Automatic Audio Content Analysis. 4th ACM International Conference on Multimedia, Boston, 18-22 November 1996, 21-30. https://doi.org/10.1145/244130.244139

[31] Pereira, F.C., Costa, H. and Pereira, N.M. (2009) An Off-Line Map-Matching Algorithm for Incomplete Map Databases. European Transport Research Review, 1, 107124. https://doi.org/10.1007/s12544-009-0013-6

[32] Hershberger, J. and Snoeyink, J. (1992) Speeding up the Douglas-Peucker LineSimplification Algorithm. 5th Symposium on Data Handling, Charleston, 3-7 August 1992, 134-143.

[33] Visvalingam, M. and Whyatt, J.D. (1993) Line Generalisation by Repeated Elimination of Points. The Cartographic Journal, 30, 46-51.

https://doi.org/10.1179/caj.1993.30.1.46

[34] Dravitzki, V.K., Jackett, R.J. and Wood, C.W.B. (2011) The Variability of Road Traffic Noise and Implications for Compliance with the Noise Conditions of Roading Designations. New Zealand Transport Agency, Wellington. 
[35] United Kingdom of Great Britain and Northern Ireland, Department of Transport, Welsh Office (1988) Calculation of Road Traffic Noise. Her Majesty's Stationery Office, London.

[36] International Organization for Standardization (2011) ISO 19156: 2011 Geographic Information-Observations and Measurements.

[37] Open Geospatial Consortium (2013) IS 12-006 Sensor Observation Service. https://portal.opengeospatial.org/files/?artifact id=47599

[38] Duda, P. (2016) Mobile Noise System Architecture. 6th International Conference on Cartography and GIS, Albena, 13-17 June 2016, 731-739.

Submit or recommend next manuscript to SCIRP and we will provide best service for you:

Accepting pre-submission inquiries through Email, Facebook, LinkedIn, Twitter, etc.

A wide selection of journals (inclusive of 9 subjects, more than 200 journals)

Providing 24-hour high-quality service

User-friendly online submission system

Fair and swift peer-review system

Efficient typesetting and proofreading procedure

Display of the result of downloads and visits, as well as the number of cited articles

Maximum dissemination of your research work

Submit your manuscript at: http://papersubmission.scirp.org/

Or contact gep@scirp.org 